Communications in Physics, Vol. 31, No. 2 (2021), pp. 219-225

DOI:10.15625/0868-3166/14942

\title{
UNFOLDING BAND AND ABSORPTION ENERGY SHIFT OF Si-Ge NANO CRYSTALS FROM FIRST-PRINCIPLES CALCULATIONS
}

\author{
TRAN VAN QUANG ${ }^{1}$, TRAN THI THUY DUONG ${ }^{2}$, LE THANH CONG ${ }^{2}$, NGO NGOC HA $^{2}$, \\ NGUYEN THI THUY NHUNG ${ }^{3}$ AND HUY-VIET NGUYEN ${ }^{3, \dagger}$
}

${ }^{1}$ University of Transportation and Communication, No.3 Cau Giay, Hanoi, Vietnam

${ }^{2}$ International Training Institute for Materials Science,

Hanoi University of Science and Technology, No. 1 Dai Co Viet road, Hanoi, Vietnam

${ }^{3}$ Institute of Physics, Vietnam Academy of Science and Technology,

18 Hoang Quoc Viet, Cay Giay, Hanoi, Vietnam

E-mail: ${ }^{\dagger}$ nhviet@iop.vast.ac.vn

Received 6 September 2020

Accepted for publication 29 December 2020

Published 22 April 2021

\begin{abstract}
Physical properties of the $S i_{1-x} G e_{x}$ alloys (x being the composition of Ge) can be understood and predicted from their electronic band structures. In this paper, electronic band structures of the $\mathrm{Si}_{1-x} G e_{x}$ alloys are calculated using the first-principles density functional theory. The supper cell approach employed in our calculations leads to folding of electronic bands into the smaller Brillouin zone of the supercell, especially at the $\Gamma$ point. This often leads to the misinterpretation that the materials have direct band gap. The problem can be resolved by an unfolding band technique which allows us to recover the primitive cell picture of band structure of $\mathrm{Si}_{1-x} G e_{x}$. As a result, unfolded electronic bands correctly show an indirect band gap with the valence band maximum (VBM) at the $\Gamma$ point and the conduction band minimum (CBM) shifted away from $\Gamma$. CBM is gradually shifted from a point along $\Gamma X$ symmetry line (associated with $\mathrm{Si}$ ) to the $L$ point (associated with Ge) with the increased Ge composition $x$ and the switching occurs at $x$ in the range of 0.6 0.8 which is in accordance with the calculation using $\mathbf{k p}$ method. Moreover, the additional electron pockets appear and develop at $\Gamma$ and $L$. This provides more comprehensive understanding for our recent experimental observations on the shift of the absorption energy assigned to $E 1$ direct transitions within $L$ and $\Gamma$ points in the Brillouin zone of $S i_{1-x} G e_{x}$ alloy nanocrystals.
\end{abstract}

Keywords: DFT-GGA, SiGe alloys, nanocrystals, unfolding band.

Classification numbers: 31.15.A-; 71.15.Mb; 72.20.Pa; 84.60.Rb; 71.20.-b.

(C)2021 Vietnam Academy of Science and Technology 


\section{INTRODUCTION}

The binary alloys of silicon $(\mathrm{Si})$ and germanium $(\mathrm{Ge}), \mathrm{Si}_{1-x} \mathrm{Ge}_{x}$ with $\mathrm{x}$ being the composition of $\mathrm{Ge}$, have been of great interest in recent years thanks to their potential applications in manufacturing novel advanced opto-electronic devices [1-7]. With respect to thermoelectricity, the $\mathrm{Si}_{1-x} \mathrm{Ge}_{x}$ alloys have attracted much attention as energy harvesting materials. Large thermoelectric figure-of-merits from $\mathrm{SiGe}$ nanowires was recently investigated experimentally and computationally [8]. Novel approaches in $\mathrm{Si}_{1-x} \mathrm{Ge}_{x}$ nanostructures for high-efficient thermoelectric devices were realized $[9,10]$. In the fundamental aspects, electrical and optical properties of the alloys are primarily determined by the band structure thereby the crystal structures [11-13]. The formation and stability of the crystalline $\mathrm{Si}-\mathrm{Ge}$ alloys can be predicted by theoretical calculations and analysis $[14,15]$.

At nanoscale, the $\mathrm{Si}_{1-x} \mathrm{Ge}_{x}$ alloys might somehow be concerned as both impurities and/or structural distortions, which are considered as perturbing agents to modify the electrical properties of the materials. Assessing how the band structure alters under these perturbations is therefore of major interest. For the actual supercell, the resulting first Brillouin zone is tiny and contains many flat-looking bands that no longer resembles the original band structures. Thus, it is not providing comprehensive information for understanding of experimental data. The information is now hidden in the Kohn-Sham orbitals [16], instead of the dispersion of the bands.

To excess the information and circumvent these difficulties, in this report, by explicitly utilizing these Kohn-Sham orbitals with an unfolding band technique, we present results of unfolded band structures of the first Brillouin zone of the supercell of $\mathrm{Si}_{1-x} \mathrm{Ge}_{x}$ (SBZ) back to the larger first Brillouin zone of the primitive cell (BZ). The $\boldsymbol{k p}$ calculations is also conducted and demonstrated for comparison.

\section{COMPUTATIONAL DETAILS}

We carried out first-principles calculation within density functional theory (DFT) by using the plane wave self-consistent field code as implemented in the Quantum Espresso package [17]. We used generalized gradient approximation (GGA) for treating exchange correlation energy [18]. The Non Linear Core Correction has been also invoked. To treat the $\mathrm{Si}_{1-x} \mathrm{Ge}_{x}$ alloys, as illustrated in Fig. 1, we build a supercell from doubling of the primitive face-centered cubic structure (FCC) cell (see Fig. 1b), which contain 2 atoms, in all directions of the lattice vectors to form the desired cell which contains 16 atoms (see Fig. 1(a)). $\mathrm{Si}_{1-x} \mathrm{Ge}_{x}$ is obtained by the substitution of Ge into $\mathrm{Si}$ sites. A $6 \times 6 \times 6$ Monkhorst-Pack k-point grid and 34 Ry kinetic energy cutoff for wavefunction, which were validated by the calculation of total energy as shown in Fig. 2, have been used in self-consistent calculation.

Accordingly, the replacement of $\mathrm{Ge}$ atoms from $\mathrm{Si}$ atoms in the cell composes different $\mathrm{Si}_{1-x} \mathrm{Ge}_{x}$ alloys (i.e., $\mathrm{x}=0.0625,0.1875,0.3125,0.6250$, and 0.8125 ), e.g. $\mathrm{x}=0.0625$ is for the replacement of one Ge atom into $\mathrm{Si}$ site in the 16-atoms supercell $(x=1 / 16)$ (see FIG. 1a). The total energies are calculated and fitted to the Murnaghan's equation of state [19] to estimate the equilibrium lattice constants [15]. These equilibrium lattice constants, which were found to be $5.49,5.53,5.56,5.66$, and $5.72 \AA$, are used for further calculations. The unfolding band technique is used to analyze the electronic bands along high symmetry lines which has been performed using 
the BandUP code [20-23]. Accordingly, it is noted that the first Brillouin zone of the primitive cell is 8 times larger than the first Brillouin zone of the supercell cell.
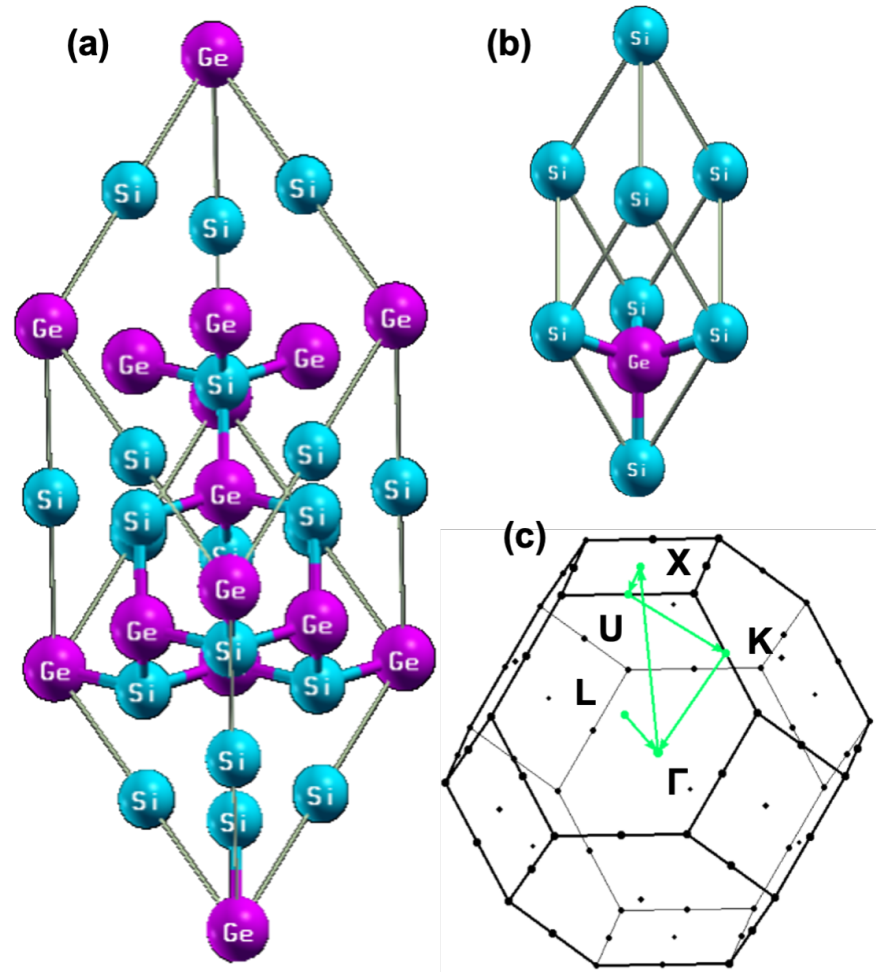

Fig. 1. The supercell of (a) $\mathrm{Si}_{1-x} \mathrm{Ge}_{x}(x=5 / 16)$ and (b) the real primitive $\mathrm{Si}_{1-x} \mathrm{Ge}_{x}$ $(x=1 / 2)$ crystal structure shown together with its Brillouin zone.

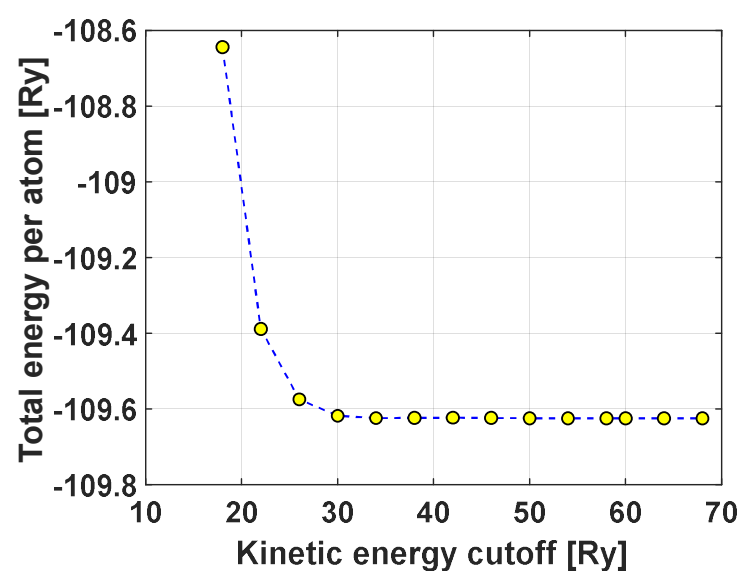

Fig. 2. Total energy per atom as a function of kinetic energy cutoff. 


\section{RESULTS AND DISCUSSION}

In Fig. 3, we presented the result of band structure of $\mathrm{Si}_{1-x} \mathrm{Ge}_{x}$ with $x=0.3125(5 / 16)$ and $x=0.625(10 / 16)$ along high symmetry lines of the Brillouin zone of supercell (SBZ). The band structure of $\mathrm{Si}_{1-x} \mathrm{Ge}_{x}$ with $x=0$ (pure $\mathrm{Si}$ ) is also presented for the comparison. Due to the period of the original cell grows longer, the size of first Brillouin zone of the supercell shrinks. In consequence, a few complicated bands appear in the SBZ. The bands are now folded. As can be seen, the valence band gradually altered with the variation of $x$ but the change is small. Accordingly, we might use the band structure of the primitive cell of $\mathrm{Si}, \mathrm{SiGe}$ and $\mathrm{Ge}$ which can be calculated routinely within DFT to analyze the valence band as shown in Ref. [24].

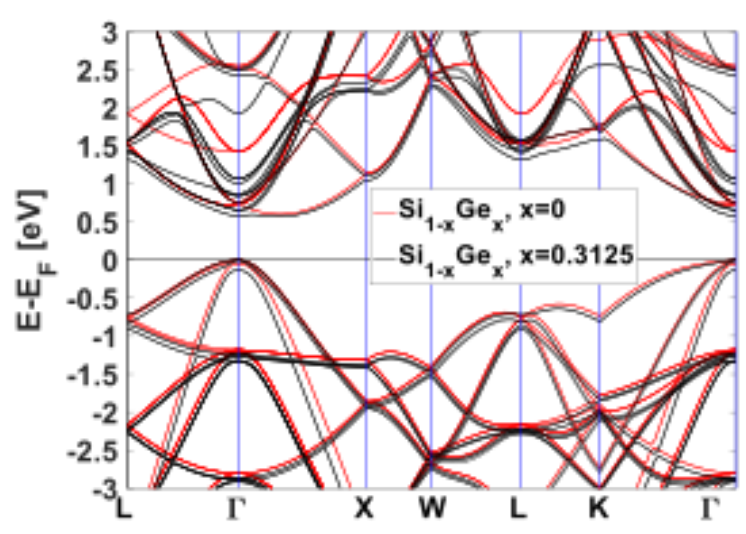

(a)

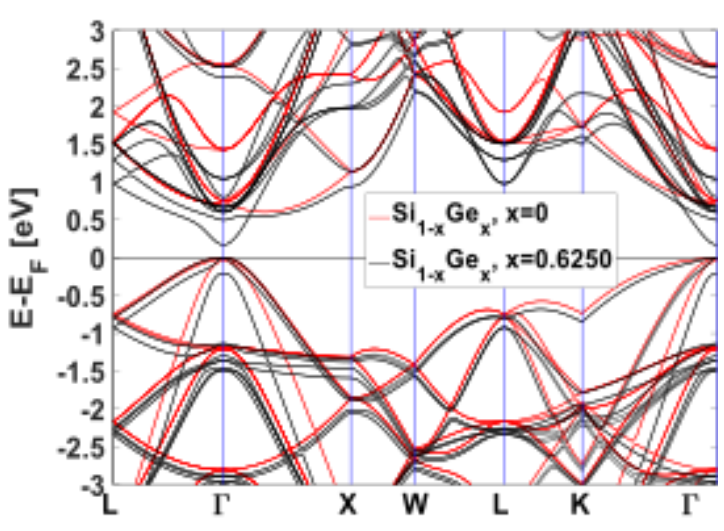

(b)

Fig. 3. (Color online) Band structure calculation along high symmetry lines of the supercell Brillouin zone of $\mathrm{Si}_{1-x} \mathrm{Ge}_{x}$ alloys for (a) $x=5 / 16$ and (b) $x=10 / 16$ shown together with band structure of $\mathrm{Si}$ for comparison.

However, the significant change was found in the conduction bands where the complicated and folded bands were observed. The conduction band minimum (CBM) of Si occurs at a point along $\Gamma X$ of SBZ. Obviously, CBM of $\mathrm{Si}$ in BZ is also found at a point along this line (see Fig. $4 \mathrm{a}$ below). We noted that SBZ and BZ have the same shape, but the size of SBZ is eight times smaller than the size of BZ. When Ge atoms are distributed, as shown in SBZ, the local band-conduction extremum at $\Gamma$ appears and gradually shifts down to reduce the band gap of the compound. This behavior occurs due to the band folded misleading to a direct semiconductor band structure. The band gap we obtained in the calculations are $0.67,0.65,0.66,0.32,0.18 \mathrm{eV}$ for $\mathrm{Si}_{1-x} \mathrm{Ge}_{x}$ with $x=1 / 16,3 / 16,5 / 16,10 / 16$, and 13/16, respectively. In turn, the exact band gaps of those should be larger due to GGA and spin-orbital coupling approximation which leads underestimates the calculated band gaps [25-27].

To analyze, we unfold the band structure by mapping bands in SBZ to those in BZ. In Fig. 4, we present our calculation of band structure of (a) $\mathrm{Si}_{1-x} \mathrm{Ge}_{x}(x=5 / 16)$ shown together with that of (b) Si along high symmetry lines in the first Brillouin zone (of the original FCC primitive cell). The color scale represents the weight function $\delta N$ as described in Ref. [23]. It somehow describes 


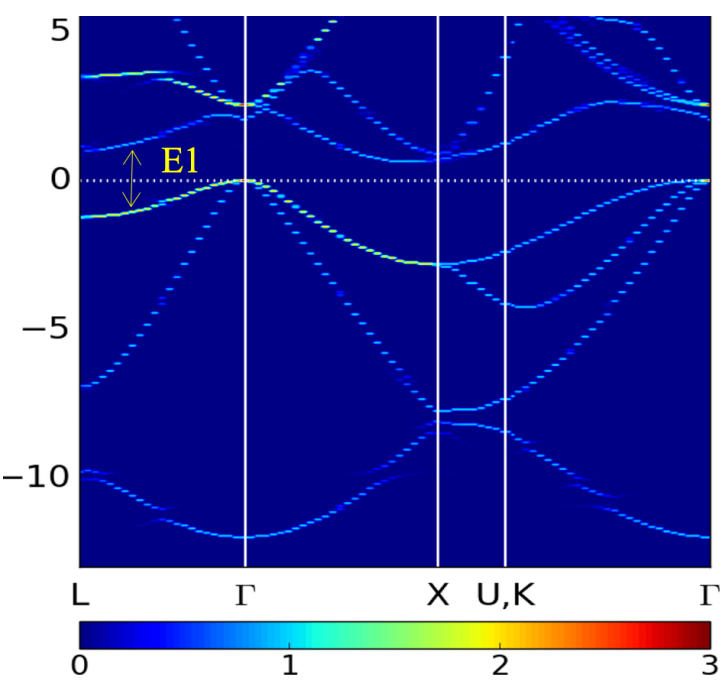

(a)

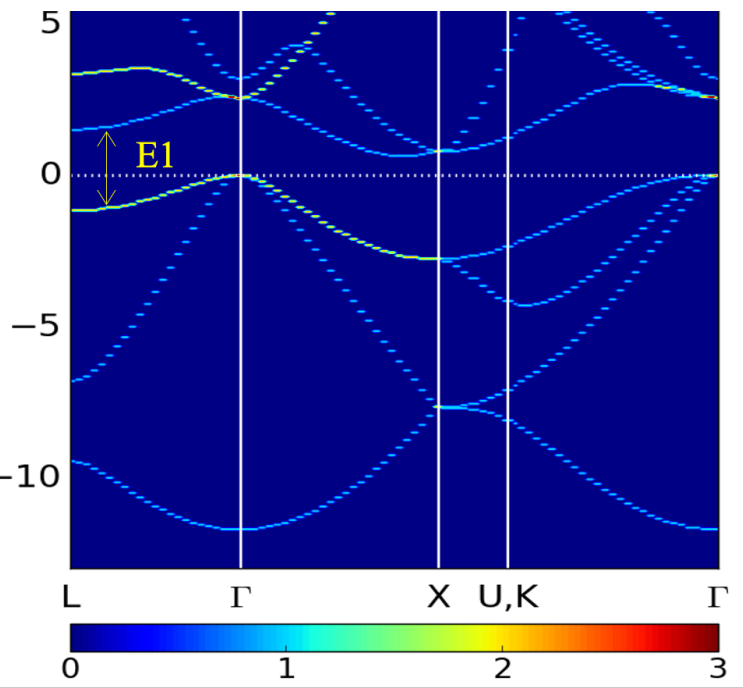

(b)

Fig. 4. (Color online) Band structure of (a) $\mathrm{Si}_{1-x} \mathrm{Ge}_{x}$ alloy (x = 5/16) shown together with that of (b) Si along high symmetry lines in the real primitive first Brillouin zone for the comparison.

the probability of electrons and/or holes occupied in a particular state determined by an energy band. The calculation of band structure of $\mathrm{Si}$ is for two purposes: for verifying the reliability of the calculation and for the comparison of other results. As can be seen, the results are exactly reproduced the band structure calculation using the real primitive cell of $\mathrm{Si}$. The $\mathrm{Ge}$ atoms enter the matrix to replace $\mathrm{Si}$, they cause the significant alternation of the band at $\Gamma$ and $L$. The second conduction band from Fermi energy is likely to be retained where the first band and the third band slowly shift to each other. At some points, they hydride and detach. As can be seen, the valley at $\Gamma$ in the conduction band gradually shows up with the increase of the Ge composition. This valley yields greater probability of electron occupation, which forms an electron pocket as n-type doping introduced.

To substantiate this point, we performed all band structure calculation for $\mathrm{Si}_{1-x} \mathrm{Ge}_{x}$ with $x=1 / 16,3 / 16,5 / 16,7 / 16,10 / 16$ and 13/16. The case of $x=10 / 16$ is illustrated in Fig. 5a. The band structure calculation using kp method [24,28] is presented in Fig. $5 \mathrm{~b}$ for the comparison. As can be seen in Fig. 5a, there are three conduction band extrema occurred at $L$, at $\Gamma$ and at a point along $\Gamma X$. Among them, regarding to the SBZ shown in Fig. 3(b), the two extrema are folded at $\Gamma$ point with two extrema at two distinguished bands, i.e. the first and the second bands from Fermi energy. And the last one occurs at a point along $\Gamma X$ which is closer to $\Gamma$ than $X$. In the SBZ scheme, $L$ point in BZ folds into $\Gamma$ point in SBZ. The CBM is gradually shifted from a point along $\Gamma X$ (associated with $\mathrm{Si}$ ) to the $\mathrm{L}$ point (associated with $\mathrm{Ge}$ ) which is folded into the $\Gamma$ point in SBZ. The switching occurs at $\mathrm{x}$ in the range of 0.60 .8 which is well consistent with the kp calculation as shown in FIG. 5b. As can be seen, the population of hole states at the highest band of valence bands is largest. And the electrons occupied in the lowest conduction band along $\Gamma-L$ is relatively large. It facilitates to promote the excitations of electrons and holes between the two states which plays 
an important role for the optical property of the alloy. The width of the transition is determined by the separation between the two states, i.e. $E 1$. Accordingly, $E 1$ is gradually reduced with the increase of $x$. These unfolded band structures could be validated by experiment of angle-resolved photoemission spectroscopy (ARPS) where the electronic band structures of the materials could be directly measured. In the current circumstance, unavailability of the facility has limited us from carrying out the ARPS experiment. Nevertheless, our theoretical results might contribute to explain the shifting of the absorption energy assigned as E1 direct transitions within $L$ and $\Gamma$ points in the Brillouin zone which was recently reported in Ref. [29].

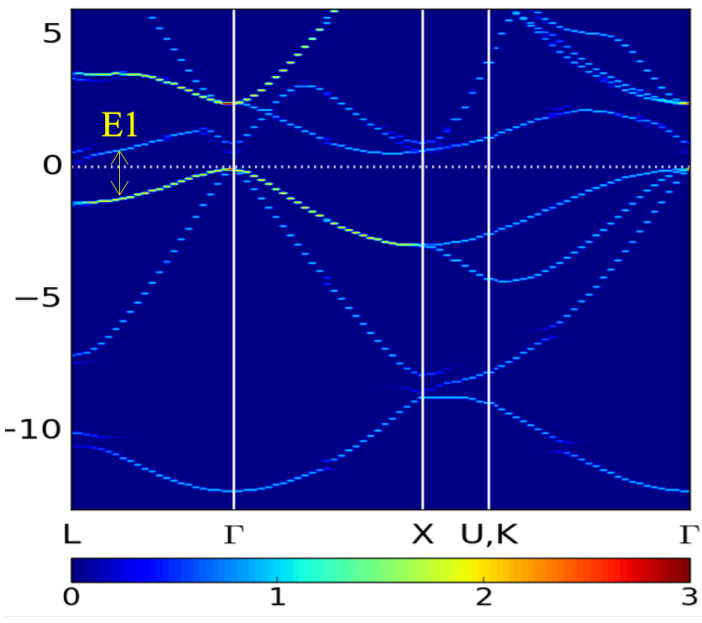

(a)

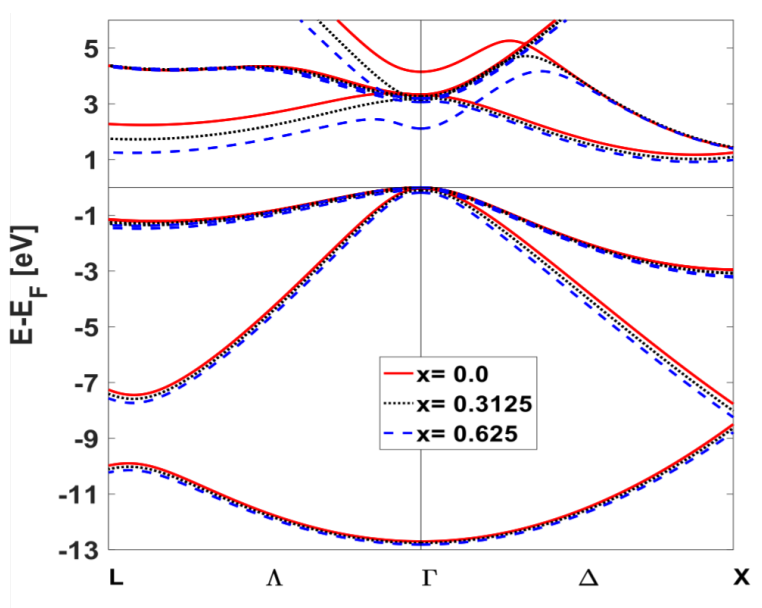

(b)

Fig. 5. (Color online) Calculated band structure along high symmetry lines of (a) $\mathrm{Si}_{1-x} \mathrm{Ge}_{x}(x=10 / 16)$ using DFT unfolding band technique and (b) $\mathrm{Si}_{1-x} \mathrm{Ge}_{x}(x=0$, 5/16 and 10/16) using $\boldsymbol{k p}$ method.

\section{CONCLUSION}

The first-principles density-functional-theory calculations was employed to study the band structure of $\mathrm{Si}_{1-x} \mathrm{Ge}_{x}$ alloys. Using the supercell approach, we found that the significant change occurred at the $\Gamma$ point in the conduction band. The increase of Ge composition leads to downward shifting of extremum to $\Gamma$ point. The primitive cell picture of band structure was recovered by unfolding band of the supercell structure to the corresponding primitive cell. In this scheme, there is a hybridization between the first and the third lowest conduction bands from Fermi energy. CBM is gradually shifted from a point along $\Gamma X$ (associated with $\mathrm{Si}$ ) to the $L$ point (associated with $\mathrm{Ge}$ ) which is folded into the $\Gamma$ point in SBZ. The switching occurs at $x$ in the range of $0.6 \sim 0.8$ which is in accordance with the calculation using $\boldsymbol{k p}$ method and the experimental observation. The reduction of the gap between the highest valence band and the lowest conduction band gives rise to the red shift of the absorption energy assigned to E1 direct transitions with the increase of $x$. The unfolding band technique could be routinely applied to compute perturbed band structures of $\mathrm{Si}-\mathrm{Ge}$ nanocrystal for further studies. 


\section{ACKNOWLEDGMENTS}

This research is funded by Vietnam-Russian academic bilateral research program, the grant number QTRU01.01/18-19.

\section{REFERENCES}

[1] S. Choi, S.H. Tan, Z. Li, Y. Kim, C. Choi, P.Y. Chen, H. Yeon, S. Yu and J. Kim, Nat. Mater. 17 (2018) 335.

[2] D. Vasilache, A. Cismaru, M. Dragoman, I. Stavarache, C. Palade, A.-M. Lepadatu and M.L. Ciurea, Phys. Status Solidi A 2132 (2016) 255.

[3] F. Meillaud, M. Boccard, G. Bugnon, M. Despeisse, S. Haenni, F.J. Haug, J. Persoz, J.W. Schuettauf, M. Stuckelberger, C. Ballif, Mater. Today 18 (2015) 378.

[4] M. Amato, M. Palummo, R. Rurali, S. Ossicini, E. Fondamentale, U. Paris-sud, A.P. Morselli and I.-R. Emilia, Chem. Rev. 114 (2014) 1371.

[5] X. Wang, H. Li, R. Camacho-aguilera, Y. Cai, L.C. Kimerling, J. Michel and J. Liu, Opt. Lett. 38 (2013) 652.

[6] D.M. Paskiewicz, B. Tanto, D.E. Savage and M.G. Lagally, ACS Nano 5 (2011) 5814.

[7] E.S. Zhukova, B.P. Gorshunov, V. a. Yuryev, L. V. Arapkina, K. V. Chizh, V. a. Chapnin, V.P. Kalinushkin, a. S. Prokhorov and G.N. Mikhailova, JETP Lett. 92 (2010) 793.

[8] E.K. Lee, L. Yin, Y. Lee, J.W. Lee, S.J. Lee, J. Lee, S.N. Cha, D. Whang, G.S. Hwang, K. Hippalgaonkar, A. Majumdar, C. Yu, B. L. Choi, J. M. Kim and K. Kim, Nano Lett. 12 (2012) 2918.

[9] J. Li, Q. Xiang, R. Ze, M. Ma, S. Wang, Q. Xie and Y. Xiang, Appl. Therm. Eng. 134 (2018) 266.

[10] C.P. Goyal, M. Omprakash, M. Navaneethan, T. Takeuchi, Y. Shimura, M. Shimomura, S. Ponnusamy, Y. Hayakawa and H. Ikeda, J. Phys. Commun. 3 (2019) 075007.

[11] P. Logan and X. Peng, Phys. Rev. B 80 (2009) 115322.

[12] T. Van Quang and M. Kim, J. Appl. Phys. 113 (2013) 17A934.

[13] M. V. Fischetti and S. E. Laux, J. Appl. Phys. 80 (1996) 2234.

[14] J.I. Abdul Rashid, J. Abdullah, N.A. Yusof and R. Hajian, J. Nanomater. 2013 (2013) 328093.

[15] N. T. Giang, L. T. Cong, N. D. Dung, T. Van Quang and N. N. Ha, J. Phys. Chem. Solids 93 (2016) 121.

[16] W. Kohn and L. J. Sham, Phys. Rev. 140 (1965) A1134.

[17] P. Giannozzi, S. Baroni, N. Bonini, M. Calandra, R. Car, C. Cavazzoni, D. Ceresoli, G.L. Chiarotti, M. Cococcioni, I. Dabo, A.D. Corso, S. Fabris, G. Fratesi, S. de Gironcoli, R. Gebauer, U. Gerstmann, C. Gougoussis, A. Kokalj, M. Lazzeri, L. Martin-samos, N. Marzari, F. Mauri, R. Mazzarello, S. Paolini, A. Pasquarello, L. Paulatto, C. Sbraccia, S. Scandolo, G. Sclauzero, A.P. Seitsonen, A. Smogunov, P. Umari, R.M. Wentzcovitch, S. De Gironcoli, S. Fabris, G. Fratesi, R. Gebauer, U. Gerstmann, C. Gougoussis, A. Kokalj, M. Lazzeri, L. Martin-samos, N. Marzari, F. Mauri, R. Mazzarello, S. Paolini, A. Pasquarello, L. Paulatto, C. Sbraccia, A. Smogunov and P. Umari, J. Phys. Condens. Matter 21 (2009) 395502.

[18] J. P. Perdew, K. Burke and M. Ernzerhof, Phys. Rev. Lett. 77 (1996) 3865.

[19] F. D. Murnaghan, Proc. Natl. Acad. Sci. U. S. A. 30 (1944) 244.

[20] C. C. Lee, Y. Yamada-Takamura, T. Ozaki, J. Phys. Condens. Matter. 25 (2013) 345501.

[21] W. Ku, T. Berlijn and C. C. Lee, Phys. Rev. Lett. 104 (2010) 216401.

[22] P. V. C. Medeiros, S. Stafström and J. Björk, Phys. Rev. B 89 (2014) $041407(\mathrm{R})$.

[23] P. V. C. Medeiros, S. S. Tsirkin and S. Stafström and J. Björk, Phys. Rev. B 91 (2015) 041116(R).

[24] T. Van Quang, N.T. Giang, N.N. Ha, VNU J. Sci. Math. - Phys. 32 (2016) 57.

[25] P. Haas, F. Tran, P. Blaha, Phys. Rev. B 79 (2009) 085104.

[26] T. Quang, H. Lim and M. Kim, J. Korean Phys. Soc. 61 (2012) 1728.

[27] D. M. Bylander, L. Kleinman, Phys. Rev. B 41 (1990) 7868.

[28] W. J. Elder, R. M. Ward, J. Zhang, Phys. Rev. B 83 (2011) 165210.

[29] N. N. Ha, N. T. Giang, T. T. T. Thuy, N. N. Trung, N. D. Dung, S. Saeed and T. Gregorkiewicz, Nanotechnology 26 (2015) 375701. 